\title{
COMPARATIVE STUDY OF EFFECTIVENESS AND RESISTANCE PROFILE OF CHLOROQUINE AND SULFADOXINE-PYRIMETHAMINE IN UNCOMPLICATED PLASMODIUM FALCIPARUM MALARIA IN KOLKATA
}

Ayan Basu ${ }^{1}$,Santanu Saha², Biswanath Sharma Sarkar ${ }^{3}$, Somnath Mukherjee ${ }^{4}$, Subhasish Kamal Guha5 ${ }^{5}$ Dyuti Basu 6

\section{HOW TO CITE THIS ARTICLE:}

Ayan Basu, Santanu Saha, Biswanath Sharma Sarkar, Somnath Mukherjee, Subhasish Kamal Guha, Dyuti Basu. "Comparative study of Effectiveness and Resistance profile of Chloroquine and Sulfadoxine - Pyrimethamine in Uncomplicated Plasmodium Falciparum Malaria in Kolkata".Journal of Evolution of Medical and Dental Sciences 2014; Vol. 3, Issue 02, January 13; Page: 246-256, DOI:10.14260/jemds/2014/1817

ABSTRACT: Malaria is one of the major public health problems of the country. Factors responsible for re- emergence of malaria in India was due to emergence and spread of chloroquine resistant Plasmodium falciparum strains across the country coupled with steady rise in insecticide resistance of the vector mosquitoes. Very little is known about the drug resistance status of P. falciparum in India. As per National Vector Borne Diseases Control Programme (NVBDCP), chloroquine is the drug of choice for uncomplicated P. falciparum cases and the combination of Artesunate and SulfadoxinePyrimethamine(SP) is being used to treat the documented chloroquine resistant uncomplicated cases. To evaluate the comparative effectiveness and resistance profile of Chloroquine vis-à-vis Sulfadoxine-Pyrimethamine (SP) in uncomplicated Plasmodium falciparum cases as the first line therapy a study was undertaken at the Malaria Clinic of School of Tropical Medicine, Kolkata during the period from July 2008 to January 2009 under Ward no. 44 of Kolkata Municipal Corporation. Following WHO protocol 2003 and WHO guideline 2006, a total of 100 parasitologically confirmed Plasmodium falciparum cases were recruited as per the recruitment criteria. Among them, 50 patients were given Chloroquine and another 50 patients were given SP. Eight patients were excluded or lost to follow-up during the follow-up period because of failure to follow the protocol.It was observed that in the chloroquine group out of 50 patients, $30(60 \%)$ showed Adequate Clinical and Parasitological Response (ACPR), 15 (30\%) had Late Treatment Failure (LTF) and remaining 5 $(10 \%)$ were lost during the follow up period (LFU). On the other hand in the SP group out of 50 patients, 46 (92\%) showed ACPR and only one (2\%) had LTF and 3 patients were LFU. The difference of LTF in Chloroquine and Sulfadoxine-pyrimethamine groups was statistically significant ( $p$ value $<0.05$ ).Also there was statistically significant difference of the mean Parasite clearance time (PCT) of Chloroquine (82.7 hours) and Sulfadoxine-pyrimethamine group(61.3 hours).Chloroquine failure rate was high which was well above the WHO recommended cut off threshold for drug policy change ( $>10 \%)$, Sulfadoxine- Pyrimethamine can be used in place of Chloroquine as the first line drug in uncomplicated P. falciparum cases.

KEY WORDS: Plasmodium falciparum, Chloroquine, Sulfadoxine- Pyrimethamine, efficacy and drug resistance.

INTRODUCTION: Malaria is the most important of the parasitic diseases of humans, with transmission in 107 countries containing 3 billion people, infecting approximately $5 \%$ of the world's population and causing 1-3 million deaths each year. Malaria is one of the major public health problems of the country. Around 1.5 million laboratory confirmed cases of malaria are reported in 
the country annually. Out of the total malaria cases, $40-50 \%$ is Plasmodium falciparum.About 1.785 million cases of malaria (including 0.839 million P.falciparum cases) and 1708 deaths were reported from the country in 2006. Recently 1.525 million cases of malaria (including 0.756 million Plasmodium falciparum cases) and 935 deaths were reported in 2008 (Provisional data given by NVBDCP in Feb 2009). ${ }^{1}$

Major factors responsible for re- emergence of malaria in India was due to emergence and spread of chloroquine resistant Plasmodium falciparum strains across the country coupled with steady rise in insecticide resistance of the vector mosquitoes. The first evidence of chloroquine (CQ) resistant P.falciparum was noted in India in 1973 from Diphu area of Karbi Anglong district of Assam. Several reports of resistance were subsequently confirmed from Arunachal Pradesh, Andhra Pradesh, Assam, Chhatisgarh, Goa, Gujarat, Jharkhand, Madhya Pradesh, Maharashtra, Meghalaya, Mizoram, Nagaland, Orissa, Rajastan, Tripura, Uttar Pradesh, Karnataka, West Bengal, and Andaman Nicobar Islands. In West Bengal, during 1982-1988, Pandeya et al. (1991) ${ }^{2}$ reported one R-III focus in Purulia and two R-III foci in the district of Jalpaiguri. In recent years multi-drug resistant P. falciparum malaria has also been reported from various countries. Very little is known about the drug resistance status of P. falciparum in India. As per National Vector Borne Diseases Control Programme (NVBDCP), chloroquine is the drug of choice for uncomplicated P.falciparum cases and the combination of Artesunate and sulfadoxine-pyrimethamine is being used to treat the documented chloroquine resistant uncomplicated cases. There has been a steady rise in the proportion of recurrent P. falciparum cases (following use of recommended doses of chloroquine) attending the Malaria Clinic of School of Tropical Medicine, Kolkata $32.7 \%$ in 2001 and 66.5\% in 2005). A significant proportion of recurrent P. falciparum cases are due to chloroquine resistance (Biswas S., 2003)3. With continued use of chloroquine as first line of therapy, the number of people with RI resistance and consequently the parasite burden in the community are also increasing. This results in increased transmission of P. falciparum in the community. Recently deaths due to P. falciparum malaria have been recorded in Kolkata, foothills of Purulia and certain Tea Estates of Dooars area of Jalpaiguri (Government data). While chloroquine remains the first line drug for uncomplicated P. falciparum malaria as per NVBDCP, a section of doctors, in private as well as in Government sector, are using the Artemisinin derivatives and Quinine indiscriminately to treat uncomplicated P. falciparum malaria cases. Injudicious use of such reserved anti-malarial drugs might lead to development of rapid resistance against them. In endemic areas it's difficult to differentiate "recrudescence" and "re-infection" clinically and/or parasitologically.

This prompted us to undertake a pilot study to evaluate the effectiveness and resistance profile of chloroquine vis-à-vis sulfadoxine-pyrimethamine in uncomplicated Plasmodium falciparum cases as the first line therapy.

\section{PATIENTS AND METHODS:}

Study sites:The present study was undertaken at the Malaria Clinic of Calcutta School of Tropical Medicine, Kolkata during the period from July 2008 to January 2009 under Ward no. 44 of Kolkata Municipal Corporation, Kolkata.

Patients:A total of 100 patients were enrolled in this study (50 patients each in Chloroquine and Sulfadoxine pyrimethamine group).The patients of confirmed Plasmodium falciparum malaria from 
Malaria Clinic were randomly screened and finally recruited for the study using following inclusion and exclusion criteria.

\section{Inclusion criteria}

a. Patients belonging to ward no. 44, Kolkata Municipal Corporation

b. Of either sex and above the age of six months

c. Microscopically proved cases of Plasmodium falciparum (monoinfection)

d. Parasite density between $1,000-100,000 / \mu \mathrm{L}$ of blood.

e. Axillary temperature of $\geq 37.5^{\circ} \mathrm{C}$ or history of fever in previous 24 hours

f. Ability to follow up visits and easy access to health facilities

g. Informed consent of the patient/parent/guardian

\section{Exclusion criteria}

a. History of taking antimalarials within 15 days preceding the illness

b. Presence of mixed infection

c. Inability to provide informed consent

d. Appearance of any of the criteria of severe or complicated malaria during the present illness

e. Pregnancy and history of amenorrhoea

f. Patients with sulfonamide hypersensitivity and known G6PD deficiency

Treatment \& follow up: Study design was done as per WHO protocol 2003 and WHO guideline 2006. This protocol consists of recording essential patient information, clinical assessment, axillarytemperature, parasitemia, bodyweight on day 0 (prior to treatment) and with the stipulated drug, clinicalassessment with examination of axillary temperature on Days 1, 2, 3, 7, 14, 21 \& 28 and parasitological examination on Days 2, 3, 7, 14, 21 \&28. On Day 1 or any other day the patient was also examined for parasitemia, if he/she had any danger sign or clinical deterioration. Both thick and thin smears were taken in the same slide from finger pricked blood sample, were stained with Giemsa stain and examined under oil immersion lens microscope on Day 0, 2, 3, 7, 14, 21, 28 and on any unscheduled day.
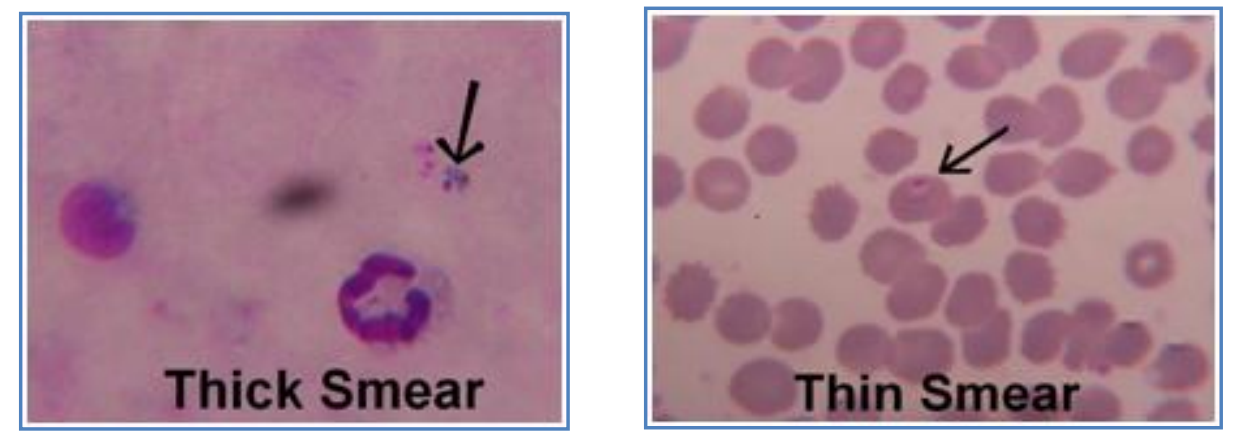

(Figure: Thick \& thin blood smear showing Giemsa stained Plasmodium falciparum ring under oil immersion with $100 \mathrm{X}$ magnification) 
Parasite load was measured by counting number of asexual forms of Plasmodium falciparum parasites against 200 leucocytes present in stained thick blood smear. In case of low parasitemia (less than 10/200 leucocytes), counting was done against 500 leucocytes. The parasite load was calculated by applying the following formula.

Parasitemia (per micro-litre) $=$ Number of parasites $\times 8000 /$ Number of leukocytes

As per National Drug Policy on Malaria (2008) by National Vector Borne Disease Control Programme (NVBDCP) the following dosage schedule was used:

\begin{tabular}{|c|c|c|c|c|c|}
\hline \multirow{3}{*}{$\begin{array}{l}\text { Age in } \\
\text { years }\end{array}$} & \multicolumn{3}{|c|}{ Day 1} & \multirow{3}{*}{$\begin{array}{c}\text { Day } 2 \\
\text { Chloroquine } \\
\text { Tablet } \\
\text { (150 mg base) }\end{array}$} & \multirow{3}{*}{$\begin{array}{c}\text { Day } 3 \\
\text { Chloroquine } \\
\text { Tablet } \\
\text { (150 mg base) }\end{array}$} \\
\hline & \multirow{2}{*}{$\begin{array}{c}\text { Chloroquine } \\
\text { Tablet } \\
\text { (150 mg base) }\end{array}$} & \multicolumn{2}{|c|}{ Primaquine } & & \\
\hline & & (Base in $\mathrm{mg}$ ) & $\begin{array}{c}\text { No. of tablets } \\
(7.5 \mathrm{mg})\end{array}$ & & \\
\hline$<1$ & $1 / 2$ & NIL & 0 & $1 / 2$ & $1 / 4$ \\
\hline $1-4$ & 1 & 7.5 & 1 & 1 & $1 / 2$ \\
\hline $5-8$ & 2 & 15 & 2 & 2 & 1 \\
\hline $9-14$ & 3 & 30 & 4 & 3 & $1 \frac{1}{2}$ \\
\hline$\geq 15$ & 4 & 45 & 6 & 4 & 2 \\
\hline
\end{tabular}

\begin{tabular}{|c|c|c|c|c|c|}
\hline \multirow[b]{2}{*}{$\begin{array}{l}\text { Age in } \\
\text { years }\end{array}$} & \multicolumn{3}{|c|}{ Sulfadoxine + Pyrimethamine(on Day 1) } & \multicolumn{2}{|c|}{ Primaquine(on Day 1) } \\
\hline & $\begin{array}{l}\text { Sulfadoxine } \\
\text { (mg base) }\end{array}$ & $\begin{array}{l}\text { Pyrimethamine } \\
\text { (mg base) }\end{array}$ & $\begin{array}{c}\text { No. of tablets } \\
(500 \mathrm{mg} \text { SDP }+25 \mathrm{mg} \\
\text { Pyrimethamine })\end{array}$ & (Base in $\mathrm{mg}$ ) & $\begin{array}{c}\text { No. of } \\
\text { tablets } \\
\text { (7.5mg) }\end{array}$ \\
\hline$<1$ & 125 & 6.25 & $1 / 4$ & NIL & 0 \\
\hline $1-4$ & 500 & 25 & 1 & 7.5 & 1 \\
\hline $5-8$ & 750 & 37.5 & $11 / 2$ & 15 & 2 \\
\hline $9-14$ & 1000 & 50 & 2 & 30 & 4 \\
\hline$\geq 15$ & 1500 & 75 & 3 & 45 & 6 \\
\hline
\end{tabular}

Classification of therapeutic response according to WHO Protocol 2003 and WHO guideline 2006

There are three categories of therapeutic responses, namely 'Early Treatment Failure' (ETF), 'Late Treatment Failure' (LTF) and 'Adequate Clinical and Parasitological Response (ACPR)'. These are defined as follows:

Early Treatment Failure (ETF) if the patient develops one of the four conditions during the first three days of follow up.

- Development of danger signs or severe malaria on Day 1, Day 2 or Day 3, in the presence of parasitemia

- Parasitemia on Day 2 higher than Day 0 count irrespective of axillary temperature 
- Parasitemia on Day 3 with axillary temperature $\geq 37.5$ degree centigrade

- Parasitemia on Day 3 greater than or equal to $25 \%$ of the count on Day 0

Late Treatment Failure (LTF) is of 2 types:

1) Late Clinical Failure (LCF)

- Development of danger signs or severe malaria after Day 3 in presence of parasitemia, without previously meeting any criteria of Early Treatment Failure

- Presence of parasitaemia and axillary temperature $\geq 37.5^{\circ} \mathrm{C}$ on any day from Day 4 to Day 28, without previously meeting any of the criteria of Early Treatment Failure

2) Late Parasitological Failure (LPF)

Presence of parasitaemia on any day from Day 7 to Day 28 and axillary temperature $<37.5$ ${ }^{\circ} \mathrm{C}$, without previously meeting any of the criteria of Early Treatment Failure or Late Clinical Failure

Adequate Clinical and Parasitological Response (ACPR)

Absence of parasitaemia on Day 28 irrespective of axillary temperature without previously meeting any of the criteria of Early Treatment Failure or Late Clinical Failure or Late Parasitological Failure

Fever Clearance Time (FCT)

This is the time from beginning of antimalarial treatment until the patient isapyrexial.This is of two types - FCTa and FCTb. FCTa is when temperature first falls below $37.5^{\circ} \mathrm{C}$ (i.e. $99.5{ }^{0} \mathrm{~F}$ ) and FCTb is when the temperature falls and remains below $37.5^{\circ} \mathrm{C}$ for 24 hours. In this study FCTa has consistently been taken into consideration.

\section{Parasite Clearance Time (PCT)}

It is the time between beginning the anti-malarial treatment and the first negative blood slide.

RESULTS:In the present study a total of 100 parasitologically confirmed Plasmodium falciparum cases belonging to ward no 44 under Kolkata Municipal Corporation were recruited during the period from July 2008 to January 2009 as per the recruitment criteria. Among them, 50 patients were given chloroquine and another 50 patients were given sulfadoxine-pyrimethamine. Eight patients were excluded or lost to follow-up (five patients in the chloroquine arm and 3 patients in the sulfadoxine-pyrimethamine arm) during the follow-up period because of failure to follow the protocol (antimalarial treatment administered by themselves or a third party) or failure to come for follow-up on the scheduled days.

There were 87 male patients (87\%) and 13 female patients (13\%). In the chloroquine arm there were $42(84 \%)$ male patients and 8 (16\%) female patients and in the sulfadoxinepyrimethamine arm there were 45 male patients (90\%) and $5(10 \%)$ female patients.

The mean age at presentation was 32.4 years (Range 10-62, Median 30). For chloroquine arm the mean age was 33.3 years (Range 10-62, Median 32.5) and for sulfadoxine-pyrimethamine arm the mean age was 31.5 years (Range 13-62, Median 29.5). 
According to the study protocol, Plasmodium falciparum parasitemic patients with fever of $>37.5^{\circ} \mathrm{C}$ (or $>99.5^{\circ} \mathrm{F}$ ) or with history of fever within the previous $24 \mathrm{hrs}$. were enrolled. On Day 0 , the mean temperature in the Chloroquine group was $100.56^{\circ} \mathrm{F}$ (range $96-103.6^{\circ} \mathrm{F}$ ) and that of Sulfadoxine-pyrimethamine group was $100.63^{\circ} \mathrm{F}$ (range 96.8-103.80 F). Six patients were afebrile at day 0; 4 in the Chloroquine group and 2 in the Sulfadoxine-pyrimethamine group. All of them had a history of fever within the previous 24 hours.

The comparison of Fever Clearance Time (FCT) of both Chloroquine and Sulfadoxinepyrimethamine arm was given in the Table 1 below.

\begin{tabular}{|c|c|c|c|}
\hline $\begin{array}{c}\text { Parameters } \\
\text { (in hours) }\end{array}$ & $\begin{array}{c}\text { All patients } \\
\left(\mathrm{N}=93^{*}\right)\end{array}$ & Chloroquine arm & Sulfadoxine-pyrimethamine arm \\
\hline Mean & 29.9 & 28.8 & 31 \\
\hline Median & 24 & 24 & 24 \\
\hline Range & $24-72$ & $24-72$ & $24-72$ \\
\hline Standard deviation & +14.001 & +13.145 & +14.818 \\
\hline The difference of mean FCT of chloroquine and sulfadoxine-pyrimethamine \\
group was not statistically significant (p value = 0.451832) \\
Table No 1: FEVER CLEARANCE TIME (FCT) \\
\hline
\end{tabular}

*Seven patients ( 5 in the chloroquine arm and 2 in the sulfadoxine-pyrimethamine arm), afebrile on the diagnosis, have been excluded for this analysis.

On day 0 , the mean parasite count of Chloroquine group was 10409.6 per $\mu \mathrm{L}$ (range 1040 $95840 \mu \mathrm{L}$ ) and that of Sulfadoxine-pyrimethamine group was 9984.04 per $\mu \mathrm{L}$ (range 1040-72000 $\mu \mathrm{L})$

The comparison of Parasite Clearance Time (PCT) of both Chloroquine and Sulfadoxinepyrimethamine arm was given in Table 2 below.

\begin{tabular}{|c|c|c|c|}
\hline $\begin{array}{c}\text { Parameters } \\
\text { (in hours) }\end{array}$ & $\begin{array}{c}\text { All patients } \\
\left(\mathrm{N}=94^{*}\right)\end{array}$ & Chloroquine arm & Sulfadoxine-pyrimethamine arm \\
\hline Mean & 72 & 82.7 & 61.3 \\
\hline Median & 72 & 72 & $48-168$ \\
\hline Range & $48-168$ & $48-168$ & +25.423 \\
\hline Standard deviation & +35.195 & +40.287 & The \\
\hline \multicolumn{2}{|r|}{ The difference of mean PCT of chloroquine and sulfadoxine-pyrimethamine } \\
group was statistically significant (p value = 0.002648)
\end{tabular}

*Six patients ( 3 in each of the chloroquine and sulfadoxine-pyrimethamine arm), lost during the follow up period, have been excluded for this analysis. 
The comparison of treatment response in both Chloroquine and Sulfadoxine-pyrimethamine arm was given in Table 3 and pie chart below.

\begin{tabular}{|c|c|c|}
\hline Treatment Response & Chloroquine arm (N=50) & $\begin{array}{c}\text { Sulfadoxine- } \\
\text { pyrimethamine arm } \\
(\mathrm{N}=50)\end{array}$ \\
\hline $\begin{array}{c}\text { Adequate Clinical and } \\
\text { Parasitological Response } \\
\text { (ACPR) }\end{array}$ & 30 patients (60\%) & 46 patients (92\%) \\
\hline $\begin{array}{c}\text { Late Treatment Failure } \\
\text { (LTF) }\end{array}$ & $\begin{array}{c}15 \text { patients (30 \%)(of which 9 patients had } \\
\text { actually Late Clinical Failure and 6 had } \\
\text { Late Parasitological Failure) }\end{array}$ & $\begin{array}{c}1 \text { patient (2 \%) (actually } \\
\text { Late Parasitological } \\
\text { Failure) }\end{array}$ \\
\hline Lost during follow up & 5 patients (10\%) & 3 patients (6\%) \\
\hline \multicolumn{2}{c}{ Table 3: TREATMENT RESPONSE } \\
\hline
\end{tabular}
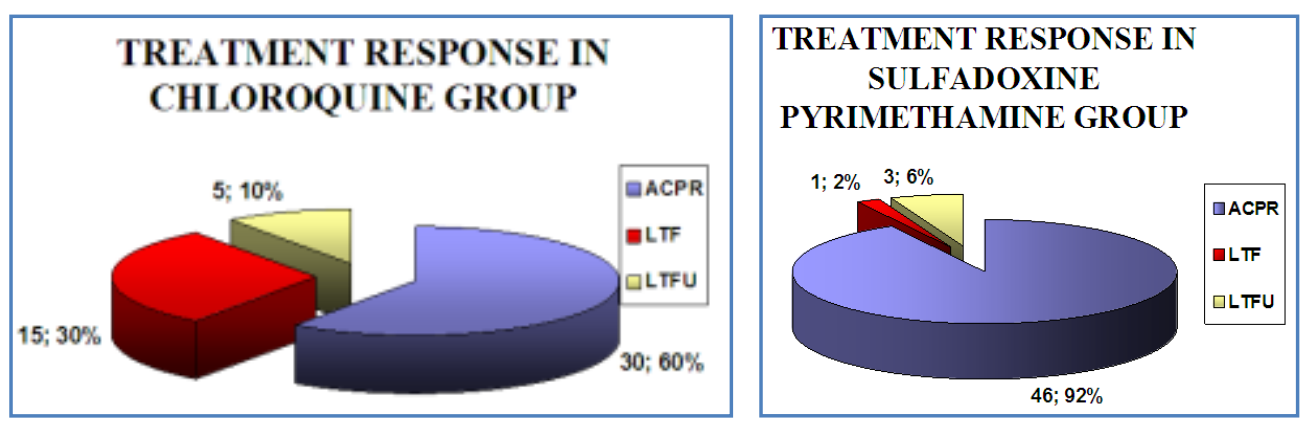

This difference of LTF was significant ( $\mathrm{p}$ value $<0.05$ ).

\section{DISCUSSION:}

Chloroquine resistance status: In our study, it was observed that in the chloroquine group out of 50 patients, 30 (60\%) showed Adequate Clinical and Parasitological Response (ACPR), 15 (30\%) had Late Treatment Failure (LTF) and remaining 5 (10\%) were lost during the follow up period.

Similar studies with Chloroquine conducted in different parts of the world showed varied results. Those are described below:

Kulkarni et al ${ }^{4}$ (2000) reported 62.5\% LTF cases in Mumbai.Ghosh et al ${ }^{5}$ in Madras showed 5 resistance cases out of 6 samples and in Jabalpur district, Madhya Pradesh 12 (85.7\%) resistance cases out of 14 samples. T Das Khatri ${ }^{6}$ in 1991 reported 24\% ACPR (out of 98 cases), 54\% R I, 4\% R II and 9\% R III in Rajasthan. In West Bengal, Pandeya et al ${ }^{2}$ (1991) showed three R III foci - two in Jalpaiguri and one in Purulia districts. The studies showed a parasite clearance of 40 per cent and 32 per cent within seventh day in Purulia and Jalpaiguri districts respectively. Biswas S ${ }^{3}$ (2005) reported 30\% ACR, 54\% LFT \& 16\% ETF in chloroquine treated cases from School of Tropical medicine, Kolkata. Recently, a study has been carried out from School of Tropical Medicine, Kolkata by Maji A. \& Guha S. K. et al ${ }^{7}$ (unpublished data) on status of antimalarial drug resistance of Plasmodium falciparum malaria in Uttar Latabari BPHC, Kalchini block \& Dhumpara PHC, Nagrakata block in the district of Jalpaiguri in 2007-08. In Kalchini Block, the treatment failure rate of Chloroquine was $59 \%$ ( ETF $11.3 \%$ \& LTF $47.7 \%$ cases) and the ACPR was $41 \%$. In Dhumpara, 
Nagrakata Block, the treatment failure rate of Chloroquine was 73\% (ETF 14.6\% \& LTF 58.4\% cases) and ACPR rate was 27\%.In Pakistan, Khan et al 8 in 2004 reported up to $16-62 \%$ chloroquine resistant Plasmodium falciparum cases. Ridwanur Rahman et al ${ }^{9}$ reported 56\% ETF cases from Bangladesh in 1996-97.Maguire et al (2002) 10 in Central Java, Indonesia reported 36 (47\%) treatment failures.Checchi et al ${ }^{11}$ in Harper, south-west Liberia reported chloroquine failure rate of 84.0\% (95\% CI 70.9-92.8\%).Moses et al ${ }^{12}$ in Kampala, Uganda showed 54\% clinical failure and 72\% parasitological failure. Fever clearance at day 3 was $85 \%$.

Mahapatra et al 13in Changlang and Lohit districts of Arunachal Pradesh recorded 23.8\% ETF, 14.3 \% LCF, 10.7 \% LPF and 51.2 \% ACPR. Schwobel et al ${ }^{14}$ in Lao PDR showed $44.8 \%$ early or late treatment failure.Checchi et al ${ }^{15}$, in Sierra Leone, showed chloroquine failure proportions were ranging from 39.5\% in Kabala to 78.8\% in Kailahun. Early failures under CQ were frequent.

Sulfadoxine-pyrimethamine resistance status:In the present study, out of 50 patients in the Sulfadoxine-pyrimethamine(SP) group, 46 (92\%) showed Adequate Clinical and Parasitological Response (ACPR) and only one (2\%) had Late Treatment Failure (LTF) and 3 patients (6\%) were lost during follow up period.

In Pakistan, Khan et al ${ }^{8}$ showed four to $25 \%$ of cases were resistant to sulfadoxinepyrimethamine. A recent study in 2007-08 by Maji A. \& Guha S. K. et al 7 (unpublished data) documented that in Kalchini Block, Jalpaiguri, the treatment failure rate of SulfadoxinePyrimethamine was $19.5 \%$ (ETF 13\% \& LTF 6.5\%) and the ACPR was 80.5\%. In Nagrakata Block of the same district, the treatment failure rate of Sulfadoxine-Pyrimethamine was 12\% (ETF 2\% \& LTF $10 \%$ ) and the ACPR was $88 \%$.Bijil et al ${ }^{16}$ (2000) reported $26 \%$ sulfadoxine-pyrimethamine resistant cases in Africa. Maguire et al ${ }^{10}$ (2002) in Central Java, Indonesia reported $22 \%$ treatment failures to SP. Almost similar results were observed in a study of SP resistance from Tanzania by Mugittu et al. ${ }^{17}$ (2005). They had documented 50.9\% ACPR at Day 28 and 17.1\% \& 24.1\% of clinical and parasitological failure respectively after 28 days of follow up. InMalawi, Plowe et al18 (2004) documented treatment failure rate of $20 \%$ in SP treated group. Checchi et al ${ }^{11}$ in Harper, south-west Liberia reported SP failure rate of 51.5\%.In Gambia, Bojang et al. ${ }^{19}$ showed ETF was $10.68 \%$ and LTF was $10 \%$ in SP treated patients. In Gambia another study by Muller et al. ${ }^{20}$ showed ETF \& LTF in Sulfadoxine-pyrimethamine group were $17 \%$ \& $14 \%$ respectively. In Uganda, Talisuna et al 21 observed parasitological failure was $61 \%$.Checchi et al ${ }^{22}$ in western Uganda reported 37\% LTF \& 15.2\% ETF.Moses et al ${ }^{12}$ in Kampala, Uganda reported 11\% clinical failure and 30\% parasitological failure among SP treated group.Mahapatra et al ${ }^{13}$ in Changlang and Lohit districts of Arunachal Pradesh recorded 14.1\% ETF, 12.6\%LCF, 8.1\% LPF and 65.2\% ACPR. Schwobel et al ${ }^{14}$ in Lao PDR documented $17.9 \%$ early or late treatment failure. Checchi et al, in Sierra ${ }^{15}$ Leone, observed that the SP failure rate varied from $23.2 \%$ in Kabala to $46.1 \%$ in Kailahun.

From the above discussion it is evident that the Chloroquine failure rate in Ward no. 44 of Kolkata Municipality Corporation is high (30\%) which is well above $(>10 \%)$ the WHO recommended cut off threshold for drug policy change, Sulfadoxine- pyrimethamine can be used in place of chloroquine as the first line drug in uncomplicated $\mathrm{P}$. falciparum cases either alone or in combination with Chloroquine. Chloroquine failure rate was very much high in Jabalpur (85.7\%) and in Harper, Liberia (84\%) reported by Ghosh et al ${ }^{5}$ and Checchi et al ${ }^{11}$ respectively. Although the Sulfadoxine- pyrimethamine failure rate in other countries was very high but in our study in Kolkata 
it was only 2\%. Whether these high failure rates was due to recrudescence (i.e. true failure/resistance) or reinfection can only be confirmed by genetic study (PCR or DNA fingerprinting).Only 4 studies (Pandeya et al, De et al, Biswas et al and Maji \& Guha S K et al) were done in West Bengal. Again our study gives an idea about the resistance pattern of central Kolkata only. To know the resistance pattern of whole of Kolkata similar studies should be carried out in other parts of the city viz. southern, north-eastern fringe and northern parts. It's also important to carry out such studies in other malaria endemic districts of West Bengal (e.g. Jalpaiguri, Paschim Midnapur, Bankura and Purulia) for better understanding of the antimalarial drug sensitivity pattern in the state. It will be useful to establish few sentinel sites in malaria endemic districts of West Bengal to monitor the therapeutic efficacy of different anti-malarial drugs.

\section{REFERENCES:}

1. National Drug Policy on Malaria 2008, Directorate of National Vector Borne Disease Control Programme (NVBDCP)

2. Pandeya A P, Barkakty B N, Narashimham M V. "Changing response of Plasmodium falciparum to chloroquine in West Bengal during 1980-1988." Journal of communicable disease, 1991; Vol 23(2): p 103-108.

3. Biswas S. "Status of chloroquine resistant Plasmodium falciparum Malaria in Kolkata." MD Thesis. 2003.

4. Kulkarni A V, Kasturi L, Amin A, Mashankar V. "Therapy and drug resistance in malaria." Indian Journal of Pediatrics, Jan. 2000, Vol. 67 (1): p 33-34.

5. Ghosh S K, Choudhury D S, Chandrahas R K, Singh N, Ramanaiah T V, Sharma V P. "Drug resistant P. falciparum in Madras (Tamil Nadu) and district Jabalpur (Madhya Pradesh)." Indian Journal of Malariology, 1989 Jun; Vol 26(2): p 87-90.

6. Das Khatri T. "Chloroquine resistant Plasmodium falciparum malaria in Rajasthan". The Journal of the Associations of Physicians India. 1991 Dec; Vol 39(12): p 926-928.

7. Guha S K, Maji A K, Pal N, Bera D K, Das M.An unpublished Project report of "Status of Antimalarial Drug Resistance of Plasmodium falciparum Malaria in Jalpaiguri", Sept 2007-Feb 2008, Department of Health \& Family Welfare, Govt of West Bengal, p 1-20

8. Khan M A, Smego R A Jr, Razi S T, Beg M A. "Emerging drug - resistance and guidelines for treatment of malaria." Journal of the College of Physicians \& Surgeons of Pakistan, 2004 May; Vol 14(5): p 319-324.

9. M Ridwanur Rahman, Dulal Chandra Paul, Md Rashid, Ajoy Ghosh, A Mannan Bangali, M A Jalil and M Abul Faiz. "A randomized controlled trial on the efficacy of alternative treatment regimens for uncomplicated falciparum malaria in a multidrug-resistant falciparum area of Bangladesh - narrowing the options for the National Malaria Control Programme." Transactions of the Royal Society of Tropical Medicine and Hygiene, November-December 2001, Vol 95(6): p 661-667.

10. Maguire J D, Lacy M D, Sururi, Sismadi P, Krisin, Wiady I et al. "Chloroquine or sulfadoxinepyrimethamine for the treatment of uncomplicated, Plasmodium falciparum malaria during an epidemic in Central Java, Indonesia." Annals of Tropical Medicine and Parasitology, 2002, Vol 96, n 7: p 655-668. 
11. F Checchi, R Durand, S Balkan, B T Vonhm, J Z Kollie, P Biberson et al. "High Plasmodium falciparum resistance to chloroquine and sulfadoxine-pyrimethamine in Harper, Liberia: results in vivo and analysis of point mutations." Transactions of the Royal Society of Tropical Medicine and Hygiene, 2002, Nov-Dec, Volume 96 (6): p 664-669.

12. Moses R Kamya, Grant Dorsey, Anne Gasasira, Grace Ndeezi, Juliet N Babirye, Sarah G Staedke and Philip J Rosenthal. "The comparative efficacy of chloroquine and sulfadoxinepyrimethamine for the treatment of uncomplicated falciparum malaria in Kampala, Uganda." Transactions of the Royal Society of Tropical Medicine and Hygiene, Volume 95, Issue 1, January-February 2001, Pages 50-55.

13. Mahapatra P K, Prakash A, Taison K, Negmu K, Gohain A C, Namchoom N S et al. "Evaluation of chloroquine (CQ) and sulphadoxine/pyrimethamine (SP) therapy in uncomplicated falciparum malaria in Indo-Myanmar border areas." Tropical Medicine \& International Health, 2005, Vol 10(5): p 478-483.

14. Schwöbel B, Jordan S, Vanisaveth V, Phetsouvanh R, Christophel E M, Phompida S et al. "Therapeutic efficacy of chloroquine plus sulphadoxine/ pyrimethamine compared with monotherapy with either chloroquine or sulphadoxine / pyrimethamine in uncomplicated Plasmodium falciparum malaria in Laos." Tropical Medicine and International Health, 2003 Jan; Vol 8(1): p 19-24.

15. Checchi F, Roddy P, Kamara S, Williams A, Morineau G, Wurie A R et al. "Sierra Leone Antimalarial Efficacy Study Collaboration. Evidence basis for antimalarial policy change in Sierra Leone: five in vivo efficacy studies of chloroquine, sulphadoxine-pyrimethamine and amodiaquine." Tropical Medicine \& International Health, 2005 Feb; Vol 10(2): p 146-153.

16. Bijil H M, Kager J, Koetsier D W, Van Der Werf T S. "Chloroquine and sulfadoxine pyrimethamine resistant Falciparum Malaria in vivo - a pilot study in rural Zambia." Tropical Medicine \& International Health, Oct. 2000, Vol. 5 (10): p 692-695.

17. Mugittu K, Abdulla S, Falk N, Masanja H, Felger I, Mashinda H et al. "Efficacy of sulphadoxinepyrimethamine in Tanzania after two years as first-line drug for uncomplicated malaria: assessment protocol an implication for treatment policy strategies." Malaria Journal 2005, Vol. $4: \mathrm{p} 55$.

18. Plowe C V, Kublin J G, Dzinjatamala F K, Kamwendo D S, Mukadam R A, Chimpeni P et al. "Sustained clinical efficacy of sulfadoxine-pyrimethamine for uncomplicated falciparum malaria in Malawi after 10 years as first line treatment: five years prospective study." British Medical Journal, 2004; Vol 328: p 545-548.

19. Bojang K A, Schneider G, Forck S, Obaro S K, Jaffar S, Pinder M et al. "A trial of Fansidar plus chloroquine or Fansidar alone for the treatment of uncomplicated malaria in Gambian children." Transactions of the Royal Society of Tropical Medicine \& Hygiene, 1998 Jan-Feb; Vol 92(1): p 73-76.

20. Müller O, Van Hensbroek M B, Jaffar S, Drakeley C, Okorie C, Joof D et al. "A randomized trial of chloroquine, amodiaquine and pyrimethamine-sulphadoxine in Gambian children with uncomplicated malaria." Tropical Medicine \& International Health, 1996 Feb; Vol 1(1): p 124132.

21. Talisuna A O, Nalunkuma-Kazibwe A, Bakyaita N, Langi P, Mutabingwa T K, Watkins W W et al. "Efficacy of sulphadoxine-pyrimethamine alone or combined with amodiaquine or chloroquine 
for the treatment of uncomplicated falciparum malaria in Ugandan children." Tropical Medicine \& International Health, 2004 Feb; Vol 9(2): p 222-229.

22. Checchi F, Piola P, Kosack C, Ardizzoni E, Klarkowski D, Kwezi E et al. "Antimalarial efficacy of sulfadoxine-pyrimethamine, amodiaquine and a combination of chloroquine plus sulfadoxinepyrimethamine in Bundi Bugyo, Western Uganda." Tropical Medicine \& International Health, 2004 Apr; Vol 9(4): p 445-450.

23. Manson's Tropical Diseases, 22 nd \& 21st edition, Table 73.14, p 1263.

24. WHO Guidelines for treatment of malaria, 2006, p 172-175.

\section{AUTHORS:}

1. Ayan Basu

2. Santanu Saha

3. Biswanath Sharma Sarkar

4. Somnath Mukherjee

5. Subhasish Kamal Guha

6. Dyuti Basu

\section{PARTICULARS OF CONTRIBUTORS:}

1. RMO Cum Clinical Tutor, Department of Tropical Medicine, School of Tropical Medicine, Kolkata.

2. Assistant Professor, Department of General Medicine, BSMCH, Bankura.

3. Associate Professor, Department of General Medicine, BSMCH, Bankura.

4. Senior Resident, Department of General Medicine, RG Kar Medical College, Kolkata.
5. Professor, Department of Tropical Medicine, School of Tropical Medicine, Kolkata.

6. Post Graduate Trainee, Department of Gynaecology \& Obstetrics, Calcutta Medical College, Kolkata.

\section{NAME ADDRESS EMAIL ID OF THE CORRESPONDING AUTHOR:}

Dr. Ayan Basu,

46/27A, Becharam Chatterjee Road,

Behala, Kolkata - 700034,

West Bengal.

Email - ayanbasustm@gmail.com

Date of Submission: 27/12/2013.

Date of Peer Review: 28/12/2013.

Date of Acceptance: 01/01/2014.

Date of Publishing: 08/01/2014. 